\title{
The future of Robotics Technology
}

\author{
Luigi Pagliarini ${ }^{1,2} \quad$ Henrik Hautop Lund ${ }^{1}$ \\ ${ }^{1}$ Centre for Playware, Technical University of Denmark, 2800 Kgs. Lyngby, Denmark \\ ${ }^{2}$ Academy of Fine Arts of Macerata, Via Berardi, 6, 405111 Macerata, Italy \\ luigipagliarini@gmail.com
}

\begin{abstract}
In the last decade the robotics industry has created millions of additional jobs led by consumer electronics and the electric vehicle industry, and by 2020 , robotics will be a $\$ 100$ billion worth industry, as big as the tourism industry. For example, the rehabilitation robot market has grown 10 times between 2010 and 2016, thanks to advancements in rehab/therapy robots, active prostheses, exoskeletons, and wearable robotics. In short, the very next decade robotics will become vital components in a number of applications and robots paired with AI will be able to perform complex actions that are capable of learning from humans, driving the intelligent automation phenomenon. Therefore, in this paper we try to depict the direction and the fields of application of such important sector of future markets, and scientific research.
\end{abstract}

Keywords: Robotics, healthcare robotics, service robotics, AI robotics, Eco-Robotics, Robot markets.

\section{Introduction}

Most certainly, in near future, and as for biological systems, Robotics will be submitted to a selective pressure under which most of its branches and authors will change. All of that will happen because of several factors: the enormous costs of production and maintenance of such machines; because of the ecosystemic and energetic costs of the robots, which are similar if not higher than any other machine; likely due to the saturation of an already seemingly fragile market. Because of that, it is quite important to try to predict the future of intelligent machines in order to focus one's efforts on the appropriate field.

In the beginning of the millennium indeed, scientists and enterprises started to apply the binomial Robot/AI to almost all of the possible domains with the "naïve" conception that the emerging technologies could have dealt with any task. Which is, in part, true. Nevertheless, on the other side, it slowly becomes self evident that there is a set of bottlenecks which are quite hard to pass. To put it in a practical way, there are at least three sets of problems, which relegate both efficiency, and functionality of such machines to a limited domain, largely excluding the dream of a "polyfunctional" robot. Such limits are (1) Mechanical, (2) Energetic and (3) Computational. Indeed, robots' mechanics tends to reach an unsustainable degree of complexity moving from a limited to a larger set of outputs. In the very same way, the energy consumption rises in parallel to the increasing of degree of freedom of any mechanics, making the energetic battery life span of a machine quite harder, up to the a critical level of inadequacy. Finally, the Computational resources of AI have largely demonstrated to be very good at solving a single task while, on the contrary, getting proportionally less efficient when handling a larger number of possible outputs. Therefore, the goal of multi-purpose robot is to be considered unreal and, oppositely, researchers and businessmen are going for a Robotics that is pretty specialized in a single task, if not a single subtask.

Given that, it happens that the number of artefacts - that we call Robots - has been exploited to such a number that both the scientific and the business markets cannot actually absorb and, as a consequence, we are about to face a phase in which selection will become necessary. Therefore, let's now see which are the largest and maybe more promising fields, trying to distinguish their 
domain of competences with their domains of application, since the two things that are not necessarily the same.

\section{Robotics Fields}

At the moment being, the number of robotics fields is nearly uncatchable, since robot technology is being applied in so many domains that nobody is able to know how many and which they are. Such an exponential growth cannot be fully tracked and we will try to identify and discuss upon the most evident fields of application, which, as far as we, comprehend are:

- Healthcare Robotics; Robotics used in the context of patient monitoring/evaluation, medical supplies delivery, and assisting healthcare professionals in unique capacities as well as, Collaborative robots and robotics used for Prevention $(1,2,3)$.

- Medical and Surgery Robotics; Devices used in hospitals mostly for assisting surgery since they allow great precision and minimal invasive procedures $(4,5)$.

- Body-machine interfaces help amputees to feed-forward controls that detect their will to move and also receive sensorial feedback that converts digital readings to feelings (6).

- Telepresence Robotics; Act as your stand-in at remote locations it is meant to be used in hospitals and for business travellers, with the idea of saving both time and money (7).

- Cyborgs, Exoskeletons, and Wearable Robotics. Allow users to augment their physical strength, helping those with physical disabilities to walk and climb (8).

- Humanoids. Combine artificial intelligence and machine learning technologies to give robots human-like expressions and reactions (9).

- Industrial. Arms, grippers and all of the warehouse robotics used for automation of industrial processes. They are used both for saving money and speed up the productions (10).

- Housekeeping. Floors, Gardens Pools and all the Robot Cleaners (11).
- Collaborative Robots. Recently the market has been opened to Domotics and other Home and Public Spaces (e.g. Shops) Automation coordinators (12).

- Military Robotics. Drones, Navigators, Researchers, Warriors and all of the possible robotics extensions which are to be applied in spying operations and battle fields (13).

- Underwater, Flying and Self -Driving Machines. All the Robotics that deals with self-piloting in all circumstances, on earth, air and water (13).

- Space Robots. All of the Robotics used in Space missions, therefore highly resistant, expert in exploration and material data collection (14).

- Entertainment. Toys, Games and Interactive Robotics for children (15).

- Art. Most creative robotics, which don't aim at a specific functionality but follows criteria of beauty and conceptual inspiration (16).

- Environmental and Alternately powered robots use sources like solar, wind and wave energy to be powered indefinitely and open up applications in areas that are off-grid (17).

- Swarm and Microbots allow emergency responders to explore environments that are too small or too dangerous for humans or larger robots; deploying them in "swarms" compensates for their relatively limited computational ability (18).

- Robotic networks emerge and allow robots to access databases, share information and learn from one another's experience.

- Modular Robotics. Robots that can arrange themselves in pre-set patterns to accomplish specific tasks (19).

\section{Analysis}

Let us now look at all of these branches distinguishing them on two different scales.

Robotics that mainly belongs to the research domain from those that aim at application, therefore, market oriented. Fields that are proficient and uprising from those that seem declining or apparently stuck in a bottle neck. 


\section{- The Market.}

Looking at the market, the richest applications are those that belong to industrial robotics, where automation of processes is requiring more and more clever and fast robots for assembling any kind of product. No comparison, this is and apparently will be the most important robotics field for quite a while, at least. Following that, there are at least few very promising fields, Entertainment, Medical, Surgery and Housekeeping Robotics. With a completely different philosophy - few expensive vs. lots of cheap sells - they established themselves into the market in a quite persistent way. Nevertheless, even if those market slices are not saturated yet, we would say they are getting close to it, leaving little space for future improvement. We cannot say the same for Industrial Robotics.

\section{- The Research.}

At the moment, researchers are keen on a set of fields that are very interesting under the scientific point of view but, oppositely, pretty poor as market chances. They are Humanoids, Telepresence, Swarm, Microbots, Robotic Networks, Modular Robotics, and BodyMachine Interfaces. Such fields are crucial for basicresearch and, probably, something exceptional will come out of all these investigations, but for the moment being, we can't foresee a single reasonably profitable application from all of them. On the contrary, Underwater, Self -Driving Machines and, in particular, Flying Robots (e.g. Amazon's Drones, etc.) are moving towards a promising number of vends and who's impact can be more than consistent in the next decade.

\section{- The Exceptions.}

An odd man out seems to be both Space and Military Robotics. For these two quite rich fields - where the concept of research and application tend to melt - there is still a large margin of exploration and exploitation. Of course, besides any further consideration, both Space and Military application can be considered, under the economical point of view, self-sustaining since they both produce and consume for their own market.

\section{- The Upcoming.}

There are few promising fields of robotics for the early future. They are Exoskeletons, Wearable Robotics,
Healthcare and Collaborative Robotics. There is, indeed, a flow of investments both from Medical and Fashion Houses that are trying to sustain the research in these fields. Investments are motivated by marketing reasons and, sometime, by a real intent of generating a new trend in their consumers market.

\section{- Out of trend}

In its short history, Robotics is already producing leftovers. Indeed, few branches of past pretty popular Robotics are getting out of trend. They are Humanoids, Geminoids, Cyborgs, etc. Their appeal and impact on the public (and on a big slice of researchers) seems to be fading away, as if both the experimentation and the public imaginary had been saturated.

\section{- Innovations.}

At the moment, the most innovative branch is Environmental and Alternately Powered Robotics that is attracting interests from different Institutes and Industries. Anyway, few are the existing application (i.e.: toys, navigation, etc.) and they do not represent a valuable set by which we could evaluate their effective potentialities. Nevertheless, they theoretically represent a quite important goal since a big obstacle in this machine-driven society is around the energy costs, and renewable energies in Robotics, as in all other applications seems to be the unique answer.

An important answer to the energy consumption question may also be found in the branch of Neurorobotics (20) which exploits Neuromorphic computing. Here, Research is investigating the use of processes similar to (human) brain processes, which offer large scale computation at a much lower energy consumption than is currently known in any computational/robotic device.

\section{Conclusions}

In the last two decades Robotics has literally exploded, both in terms of research and applications. It has invaded the people's imaginary and almost all of the existing markets, up to the point that, on one side, we can spot at robotics news each single day and, on the other, Robotics is about to reach a market slice of 100 
Billions dollars. In this paper we tried to summarize and analyse which are the most profitable and promising branches and where to look for new horizons. It appears that Industries' Automation is the leader of such a world while a number of applications are consolidating themselves or about to emerge and to play a consistent role in Robotics research and production. They are Healthcare, Surgery, Housekeeping, Autonomous Vehicles and, in part, Entertainment. We also underlined which are the branches that seem to lose affection of markets and researches, as for example Humanoids, and those who are gaining interests, as for example Alternately Powered Robotics. It is our belief that our analysis can provide a wider view on the world of Robotics and how to approach it in the early future.

\section{References}

1. H. H. Lund, "Play for the Elderly - Effect Studies of Playful Technology," in Human Aspects of IT for the Aged Population. Design for Everyday Life. (LNCS Vol. 9194, pp 500-511, Springer-Verlag, 2015)

2. H. H. Lund, and J. D. Jessen, "Effects of short-term training of community-dwelling elderly with modular interactive tiles," GAMES FOR HEALTH: Research, Development, and Clinical Applications, 3(5), 277-283, 2014.

3. A. Okamura, M. Mataric, \& H. Christensen Panels. CCC/CRA, Roadmapping for Robotics Workshop: A Research Roadmap for Medical and Healthcare Robotics. Available at: http://www.usrobotics.us/medical-ws.html (2008).

4. M. Sood, S. W. Leichtle. Essentials of Robotic Surgery, Spry Publishing LLC, Mar 1, 2013
5. T. Lendvay, (2008). Robotic Surgery Simulation: An Unintuitive Reflection. Medical Robotics Magazine. Available at: http://medicalrobotics.blogspot.com/2008/ 10/robotic-surgery-simulation-unintuitive.html .

6. T. D. Coates, Neural Interfacing, Forging the HumanMachine Connection. Morgan \& Clayton Publishers. John D. Enderle Series Editor, (2008).

7. I. R. Nourbakhsh, Robot Futures. The MIT Press, Cambridge Massachusetts, London England (2013).

8. J. L. Pons. Wearable Robots: Biomechatronic Exoskeletons. John Wiley \& Sons Ltd. (2008).

9. S. Kajita, H. Hirukawa, K. Harada, K. Yokoi, Introduction to Humanoid Robotics. Springer (2014).

10. S. Y. Nof, Springer Handbook of Automation. SpringerVerlag (2008).

11. R. Siegwart, I. R. Nourbakhsh, D. Scaramuzza Introduction to Autonomous Mobile Robots.The MIT Press, Cambridge Massachusetts, London England (2004).

12. J. Gerhart, Home Automation and Wiring, McGraw Hill Professional (1999).

13. P. J. Springer, Military Robots and Drones: A Reference Handbook. ABC-CLIO Editor (2013).

14. R. D. Launius, H. E. McCurdy. Robots in Space: Technology, Evolution, and Interplanetary Travel. The Johns Hopkins University Press, Baltimore. (2008).

15. R. Malone. Ultimate Robot. DK Pub., 2004.

16. L. Pagliarini, H. H. Lund. The Development of Robot Art. AROB, 13th International Symposium on Artificial Life and Robotics, Oita, Japan, January 31-February 2, 2008.

17. R. Hanson. The Age of Em: Work, Love, and Life when Robots Rule the Earth. Oxford University Press. (2016).

18. S. Kernbach. Handbook of Collective Robotics: Fundamentals and Challenges. Pan Stanford Publishing. (2013).

19. K. Stay, D. Brandt, D. J. Christensen. Self-reconfigurable Robots. An Introduction. MIT Press, (2010).

20. P. Artemiadis. Neuro-Robotics: From Brain Machine Interfaces to Rehabilitation Robotics. Springer (2013). 